\title{
REMARKS ON AFFINE SPRINGER FIBRES
}

\author{
G. LUSZTIG \\ Department of Mathematics, MIT, Cambridge MA 02139, USA. \\ E-mail: gyuri@math.mit.edu \\ \|\|\|\|
}

\begin{abstract}
Let $h$ be a regular semisimple element in a complex simple Lie algebra $\mathfrak{g}$. Let $\mathfrak{t}$ be an indeterminate. We consider the "variety" of Iwahori subalgebras of $\mathfrak{g}$ tensored with the power series in $\mathfrak{t}$ which contain $\mathfrak{t}$ times $h$. This variety admits a free action of a free abelian group of rank equal to the rank of $\mathfrak{g}$.
\end{abstract}

We describe a fundamental domain for this action.

Let $G$ be a simply connected almost simple algebraic group over $\mathbf{C}$ and let $\mathfrak{g}$ be the Lie algebra of $G$. Let $B$ be a Borel subgroup of $G$, let $T$ be a maximal torus of $B$ and let $\mathfrak{t}, \mathfrak{b}$ be the Lie algebras of $T, B$. Let $\mathcal{B}$ be the variety of Borel subalgebras of $\mathfrak{g}$. For any nilpotent element $N \in \mathfrak{g}$ we set $\mathcal{B}_{N}=\{\mathfrak{b} \in \mathcal{B} ; N \in \mathfrak{b}\}$ (a Springer fibre). In [1] an affine analogue of $\mathcal{B}_{N}$ ("affine Springer fibre") was introduced. Let $F=\mathbf{C}((\epsilon), A=\mathbf{C}[[\epsilon]]$, where $\epsilon$ is an indeterminate and let $\mathfrak{g}(F)=F \otimes \mathfrak{g}$ (a Lie algebra over $F$ ), $L=A \otimes \mathfrak{g}$ (a Lie algebra over $A$ ). An element $\xi \in \mathfrak{g}(F)$ is said to be topologically nilpotent if $\lim _{n \rightarrow \infty} \operatorname{ad}(\xi)^{n}=0$ in $\operatorname{End}_{F}(\mathfrak{g}(F))$. Let $\tilde{X}$ be the set of all Iwahori subalgebras of $\mathfrak{g}(F)$; this is an increasing union of projective varieties over C. According to [1], for any regular semisimple, topologically nilpotent element $\xi \in \mathfrak{g}(F)$, the set $\tilde{X}_{\xi}=\{I \in \tilde{X} ; \xi \in I\}$ is a nonempty, locally finite union of projective varieties all of the same dimension, say $b_{\xi}$. Let $\left[\tilde{X}_{\xi}\right]$ be the set of irreducible components of $\tilde{X}_{\xi}$, a finite or countable set.

Received November 17, 2019.

AMS Subject Classification: 20G99.

Key words and phrases: Simple Lie algebra, Iwahori subalgebra, fundamental domain. Supported by NSF grant DMS-1855773. 
In the remainder of this paper, $h$ denotes a fixed regular element in $\mathfrak{t}$. Then $\epsilon h \in \mathfrak{g}(F)$ is regular semisimple, topologically nilpotent so that the affine Springer fibre $\tilde{X}_{\epsilon h}=\{I \in \tilde{X} ; \epsilon h \in I\}$ is defined. From [1, §5] we see that $b_{\epsilon h}=\nu$ where $\nu=\operatorname{dim} \mathcal{B}$. As in [1, $\left.\S 3\right]$, there is a free abelian group $\Lambda$ (see Sec. 2) of rank equal to the rank of $\mathfrak{g}$ which acts freely on $\tilde{X}_{\epsilon h}$ in such a way that the induced $\Lambda$-action on $\left[\tilde{X}_{\epsilon h}\right]$ is also free and has only finitely many orbits. In this paper we will describe a fundamental domain for the $\Lambda$-action on $\tilde{X}_{\epsilon h}$. Namely, let $\mathfrak{S}^{\prime}$ be the Steinberg variety of triples $\left(E, \mathfrak{b}_{1}, \mathfrak{b}_{2}\right)$ where $\mathfrak{b}_{1} \in \mathcal{B}, \mathfrak{b}_{2} \in \mathcal{B}$ and $E \in \mathfrak{b}_{1} \cap \mathfrak{b}_{2}$ is nilpotent. Let $\mathfrak{S}$ be the fibre at $\mathfrak{b}$ of the projection $\mathfrak{S}^{\prime} \rightarrow \mathcal{B},\left(E, \mathfrak{b}_{1}, \mathfrak{b}_{2}\right) \mapsto \mathfrak{b}_{2}$. We can identify $\mathfrak{S}$ with $\left\{\left(E, \mathfrak{b}_{1}\right) ; \mathfrak{b}_{1} \in \mathcal{B}, E \in \mathfrak{n} \cap \mathfrak{b}_{1}\right\}$. We state the following result.

Theorem 1. There is a locally closed subvariety $\tilde{\Omega}$ of $\tilde{X}_{\epsilon h}$ which is a fundamental domain for the $\Lambda$-action on $\tilde{X}_{\epsilon h}$ such that $\tilde{\Omega}$ is isomorphic to $\mathfrak{S}$.

From the theorem one can deduce some information on the representation of the affine Weyl group on the vector space $\mathbf{C}\left[\tilde{X}_{\epsilon h}\right]$ with basis $\left[\tilde{X}_{\epsilon h}\right]$ defined in [3], see Section 6.

I thank Peng Shan and Zhiwei Yun for discussions.

2. Let $U$ be the unipotent radical of $B$. Let $\mathfrak{n}$ be the Lie algebra of $U$. Let $G(F), U(F), T(F)$ be the group of $F$-points of $G, U, F$ respectively. Let $G(F)$ be the group of $F$-points of $G$. Note that $G(F)$ acts naturally on $\mathfrak{g}(F)$ by the adjoint representation $g: x \mapsto \operatorname{Ad}(g)(x)$. Let $\Lambda$ be the subgroup of $T(F)$ consisting of the elements $\chi(\epsilon)$ where $\chi$ runs over the one parameter subgroups $\mathbf{C}^{*} \rightarrow T$ (viewed as homomorphisms $F^{*} \rightarrow T(F)$ ). Let $X$ be the set of $A$-Lie subalgebras of $\mathfrak{g}(F)$ of the form $\operatorname{Ad}(g)(L)$ for some $g \in G(F)$. We shall regard $X$ as an increasing union of projective algebraic varieties over $\mathbf{C}$ as in [2, §11]. For each $L^{\prime} \in X, L^{\prime} / \epsilon L^{\prime}$ inherits from $L^{\prime}$ a bracket operation and becomes a simple Lie algebra over C. Let $\pi_{L^{\prime}}: L^{\prime} \rightarrow L^{\prime} / \epsilon L^{\prime}$ be the obvious map. Let $\mathcal{B}_{L^{\prime}}$ be the set of Borel subalgebras of $L^{\prime} / \epsilon L^{\prime}$. Now $\tilde{X}$ consists of all C-Lie subalgebra of $\mathfrak{g}(F)$ of the form $\pi_{L^{\prime}}^{-1}\left(\mathfrak{b}^{\prime}\right)$ for some $L^{\prime} \in X$ and some $\mathfrak{b}^{\prime} \in \mathcal{B}_{L^{\prime}}$. We define $\pi: \tilde{X} \rightarrow X$ by $I \mapsto L^{\prime}$ where $I \subset L^{\prime}$. Note that $g: I \mapsto \operatorname{Ad}(g) I$ is a well defined action of $G(F)$ on $\tilde{X}$ which is transitive. According to [1], $t: I \mapsto \operatorname{Ad}(t) I$ defines a free action of $\Lambda$ on 
$\tilde{X}_{\epsilon h}=\{I \in \tilde{X} ; \epsilon h \in I\}$ inducing a free action of $\Lambda$ with finitely many orbits on $\left[\tilde{X}_{\epsilon h}\right]$. Let $X_{\epsilon h}=\left\{L^{\prime} \in X ; \epsilon h \in L^{\prime}\right\}$.

If $\xi \in \mathfrak{n}(F):=F \otimes \mathfrak{n}$ then $\exp (\xi) \in U(F)$ is well defined. Let $\mathfrak{n}(F)^{\prime}=$ $\oplus_{i \in \mathbf{Z} ; i<0} \epsilon^{i} \mathfrak{n} \subset \mathfrak{n}(F)$. Let $U(F)^{\prime}=\left\{\exp (\xi) ; \xi \in \mathfrak{n}(F)^{\prime}\right\} \subset U(F)$. It is well known that any $L^{\prime} \in X$ can be written in the form $\operatorname{Ad}(t) \operatorname{Ad}(u) L$ where $t \in \Lambda, u \in U(F)^{\prime}$ are uniquely determined. Hence we have a partition $X_{\epsilon h}=\sqcup_{t \in \Lambda} X_{\epsilon h, t}$ where $X_{\epsilon h, t}=\left\{\operatorname{Ad}(t) \operatorname{Ad}(u) L ; u \in U(F)^{\prime}, \epsilon h \in \operatorname{Ad}(u) L\right\}$ is a locally closed subset of $X_{\epsilon h}$. Let $\tilde{X}_{\epsilon h, t}=\pi^{-1}\left(X_{\epsilon h, t}\right)$. This is a locally closed subset of $\tilde{X}_{\epsilon h}$. Let $\Omega=X_{\epsilon h, 1}, \tilde{\Omega}=\tilde{X}_{\epsilon h, 1}=\pi^{-1}(\Omega)$. Note that

(a) $\tilde{X}_{\epsilon h}=\sqcup_{t \in \Lambda} \operatorname{Ad}(t) \tilde{\Omega}$

as a set. Thus, $\tilde{\Omega}$ is a fundamental domain for the $\Lambda$-action on $\tilde{X}_{\epsilon h}$. Let $\omega=\left\{\mathbf{E} \in \mathfrak{n}(F)^{\prime} ; \operatorname{Ad}(\exp (\mathbf{E}))(\epsilon h) \in L\right\}$. In preparation for the proof of the theorem we will prove the following result.

Lemma 3. The map $\mathbf{E}=\epsilon^{-1} E_{1}+\epsilon^{-2} E_{2}+\epsilon^{-3} E_{3}+\cdots \rightarrow E_{1}$ is a bijection $\phi: \omega \stackrel{\sim}{\longrightarrow} \mathfrak{n}$. (Here $E_{1}, E_{2}, E_{3}, \ldots$ is a sequence of elements of $\mathfrak{n}$ with $E_{i}=0$ for large $i$.)

The equation defining $\omega$ is $\exp (\operatorname{ad}(\mathbf{E}))(\epsilon h) \in L$ that is

$$
\begin{gathered}
\epsilon h+\sum_{i \geq 1} \epsilon^{-i+1}\left[E_{i}, h\right]+(1 / 2) \sum_{i, j \geq 1} \epsilon^{-i-j+1}\left[E_{i},\left[E_{j}, h\right]\right] \\
+(1 / 6) \sum_{i, j, k \geq 1} \epsilon^{-i-j-k+1}\left[E_{i},\left[E_{j},\left[E_{k}, h\right]\right]\right]+\cdots \in L,
\end{gathered}
$$

that is

$$
\begin{aligned}
& \sum_{i \geq 2} \epsilon^{-i+1}\left[E_{i}, h\right]+(1 / 2) \sum_{i, j \geq 1} \epsilon^{-i-j+1}\left[E_{i},\left[E_{j}, h\right]\right] \\
& +(1 / 6) \sum_{i, j, k \geq 1} \epsilon^{-i-j-k+1}\left[E_{i},\left[E_{j},\left[E_{k}, h\right]\right]\right]+\cdots \in L,
\end{aligned}
$$

that is

$$
\left[E_{r}, h\right]=-(1 / 2) \sum_{i, j \geq 1, i+j=r}\left[E_{i},\left[E_{j}, h\right]\right]
$$




$$
-(1 / 6) \sum_{i, j, k \geq 1, i+j+k=r}\left[E_{i},\left[E_{j},\left[E_{k}, h\right]\right]\right]+\cdots
$$

for $r=2,3, \ldots$ In the right hand side we have $i<r, j<r, k<r$, etc. Hence if $E_{r^{\prime}}$ is known for $r^{\prime}<r$ then $\left[E_{r}, h\right]$ is a well defined element of $\mathfrak{n}$. Hence $E_{r}$ is a well defined element of $\mathfrak{n}$. (Note that $E \mapsto[E, h]$ is a vector space isomorphism $\mathfrak{n} \stackrel{\sim}{\longrightarrow} \mathfrak{n}$.)

It remains to show that $E_{r}=0$ for large $r$. For $r \geq 1$ let $\mathfrak{n}^{r}$ be the subspace of $\mathfrak{n}$ spanned by all iterated brackets of $r$ elements of $\mathfrak{n}$. (Thus, $\mathfrak{n}^{1}=\mathfrak{n}, \mathfrak{n}^{2}$ is spanned by $[a, b]$ with $a, b$ in $\mathfrak{n}, \mathfrak{n}^{3}$ is spanned by $\left.[[a, b], c]\right]$ with $a, b, c$ in $\mathfrak{n}$, etc.) Note that

(b) $E \mapsto[E, h]$ is an isomorphism $\mathfrak{n}^{r} \rightarrow \mathfrak{n}^{r}$ for any $r \geq 1$.

We show by induction on $r$ that

$$
E_{r} \in \mathfrak{n}^{r} \text { for } r=1,2, \ldots
$$

For $r=1$ this is clear. Assume now that $r \geq 2$. From (a) and the induction hypothesis we deduce that $\left[E_{r}, h\right] \in \mathfrak{n}^{r}$. Using (b) we see that for some $E^{\prime} \in \mathfrak{n}^{r}$ we have $\left[E_{r}, h\right]=\left[E^{\prime}, h\right]$, hence $\left[E_{r}-E^{\prime}, h\right]=0$, hence $E_{r}=E^{\prime}$. Thus $E_{r} \in \mathfrak{n}^{r}$, proving (c). Since $\mathfrak{n}^{r}=0$ for large $r$ we see that $E_{r}=0$ for large $r$. This completes the proof of the lemma.

4. For $E \in \mathfrak{n}$ we set $u_{E}=\exp (\mathbf{E}) \in U(F)^{\prime}$ where $\mathbf{E}=\phi^{-1}(E)$ (see Lemma 3). Note that $\operatorname{Ad}\left(u_{E}\right)(\epsilon h) \in L$. Now $\mathbf{E} \mapsto \operatorname{Ad}(\exp (-\mathbf{E})) L$ is a bijection $\psi: \omega \stackrel{\sim}{\longrightarrow} \Omega$. Hence $\psi^{\prime}:=\psi \phi^{-1}: \mathfrak{n} \rightarrow \Omega$ is a bijection. We have $\psi^{\prime}(E)=$ $\operatorname{Ad}\left(u_{E}^{-1}\right) L$. We show:

(a) Let $E \in \mathfrak{n}$ and let $L_{E}=\operatorname{Ad}\left(u_{E}^{-1}\right) L \in X$. Note that $\epsilon h \in L_{E}$. Then $\pi_{L_{E}}(\epsilon h) \in L_{E} / \epsilon L_{E}$ and $\pi_{L}(-[E, h]) \in L / \epsilon L$ correspond to each other under the Lie algebra isomorphism $\tau_{E}: L / \epsilon L \stackrel{\sim}{\longrightarrow} L_{E} / \epsilon L_{E}$ induced by $\operatorname{Ad}\left(u_{E}^{-1}\right): L \stackrel{\sim}{\longrightarrow} L_{E}$.

We must show that $A d\left(u_{E}\right)(\epsilon h)=-[E, h] \bmod \epsilon L$ or that $\operatorname{Ad}(\exp (\mathbf{E}))(\epsilon h)=$ $-[E, h] \bmod \epsilon L$ where $\mathbf{E}=\epsilon^{-1} E_{1}+\epsilon^{-2} E_{2}+\epsilon^{-3} E_{3}+\cdots$ corresponds to 
$E=E_{1}$ as in Lemma 3. Thus we must show that

$$
\begin{aligned}
& \epsilon h+\sum_{i \geq 1} \epsilon^{-i+1}\left[E_{i}, h\right]+(1 / 2) \sum_{i, j \geq 1} \epsilon^{-i-j+1}\left[E_{i},\left[E_{j}, h\right]\right] \\
& +(1 / 6) \sum_{i, j, k \geq 1} \epsilon^{-i-j-k+1}\left[E_{i},\left[E_{j},\left[E_{k}, h\right]\right]\right]+=-\left[E_{1}, h\right] \bmod \epsilon L,
\end{aligned}
$$

or that

$$
\begin{aligned}
& \sum_{i \geq 2} \epsilon^{-i+1}\left[E_{i}, h\right]+(1 / 2) \sum_{i, j \geq 1} \epsilon^{-i-j+1}\left[E_{i},\left[E_{j}, h\right]\right] \\
& +(1 / 6) \sum_{i, j, k \geq 1} \epsilon^{-i-j-k+1}\left[E_{i},\left[E_{j},\left[E_{k}, h\right]\right]\right]+\cdots \in \epsilon L .
\end{aligned}
$$

But the left hand side is actually zero, by the proof of Lemma 3. This proves (a).

From (a) we deduce:

(b) the map $\beta \mapsto \tau_{E}(\beta)$ is a bijection $\left\{\beta \in \mathcal{B}_{L} ; \pi_{L}(-[E, h]) \in \beta\right\} \rightarrow\left\{\beta^{\prime} \in\right.$ $\left.\mathcal{B}_{L_{E}} ; \pi_{L_{E}}(\epsilon h) \in \beta^{\prime}\right\}$.

Taking union over all $E \in \mathfrak{n}$ and using the bijection $\psi^{\prime}: \mathfrak{n} \rightarrow \Omega$ we deduce

(c) the map $(E, \beta) \mapsto \pi_{L_{E}}^{-1}\left(\tau_{E}(\beta)\right)$ is a bijection $\left\{(E, \beta) \in \mathfrak{n} \times \mathcal{B}_{L} ; \pi_{L}(-[E, h])\right.$ $\in \beta\} \stackrel{\sim}{\longrightarrow} \tilde{\Omega}$.

We consider the bijection

(d) $\left\{(E, \beta) \in \mathfrak{n} \times \mathcal{B}_{L} ; \pi_{L}(-[E, h]) \in \beta\right\} \rightarrow \mathfrak{S}$

given by $(E, \beta) \mapsto\left(-[E, h], \mathfrak{b}_{1}\right)$ where $\mathfrak{b}_{1} \in \mathcal{B}$ is defined by $\pi_{L}\left(\mathfrak{b}_{1}\right)=\beta$. The composition of the inverse of (d) with the bijection (c) is a bijection

(e) $\mathfrak{S} \stackrel{\sim}{\longrightarrow} \tilde{\Omega}$.

From the proof we see that the bijection (e) is an isomorphism of algebraic varieties. This proves the theorem.

5. Let $N T$ be the normalizer of $T$ in $G$ and let $W=N T / T$ be the Weyl group. For any $w \in W$ let $\mathcal{B}_{w}$ be the variety consisting of all $\mathfrak{b}_{1} \in \mathcal{B}$ such that $\left(\mathfrak{b}, \mathfrak{b}_{1}\right)$ are in relative position $w$. Note that $\mathcal{B}_{w}$ is isomorphic to $\mathbf{C}^{|w|}$ 
where $|w| \in \mathbf{N}$ is the length of $w$. Let $\mathfrak{S}_{w}=\left\{\left(E, \mathfrak{b}_{1}\right) \in \mathfrak{S} ; \mathfrak{b}_{1} \in \mathcal{B}_{w}\right\}$. The second projection $\mathfrak{S}_{w} \rightarrow \mathcal{B}_{w}$ makes $\mathfrak{S}_{w}$ into a vector bundle with fibres of dimension $\nu-|w|$. Hence $\mathfrak{S}_{w}$ is isomorphic to $\mathbf{C}^{\nu}$ as an algebraic variety. We have a partition $\mathfrak{S}=\sqcup_{w \in W} \mathfrak{S}_{w}$ (as a set) with $\mathfrak{S}_{w}$ locally closed in $\mathfrak{S}$ (the closure of $\mathfrak{S}_{w}$ in $\mathfrak{S}$ is denoted by $\overline{\mathfrak{S}_{w}}$ ). Hence we have a partition $\tilde{\Omega}=\sqcup_{w \in W} \tilde{\Omega}_{w}$ (as a set) where $\tilde{\Omega}_{w}$ corresponds to $\mathfrak{S}_{w}$ under $4(\mathrm{e})$. Note that $\tilde{\Omega}_{w}$ is isomorphic to $\mathbf{C}^{\nu}$ as an algebraic variety and that $\tilde{\Omega}_{w}$ is locally closed in $\tilde{\Omega}$. For $w \in W, t \in \Lambda$ we set $\tilde{\Omega}_{w, t}=\operatorname{Ad}(t) \tilde{\Omega}_{w}$. Using $2($ a) we see that

(a) $\tilde{X}_{\epsilon h}=\sqcup_{(w, t) \in W \times \Lambda} \tilde{\Omega}_{w, t}$

as a set, where $\tilde{\Omega}_{w, t}$ is locally closed in $\tilde{X}_{\epsilon h}$ and is isomorphic to $\mathbf{C}^{\nu}$. Let $\overline{\tilde{\Omega}_{w, t}}$ be the closure of $\tilde{\Omega}_{w, t}$ in $\tilde{X}_{\epsilon h}$. Note that $\tilde{\Omega}_{w, t}$ is open dense in $\overline{\tilde{\Omega}_{w, t}}$. Since $\tilde{X}_{\epsilon h}$ is of pure dimension $\nu$, we see that

(b) $(w, t) \mapsto \overline{\tilde{\Omega}_{w, t}}$ is a bijection $W \times \Lambda \stackrel{\sim}{\longrightarrow}\left[\tilde{X}_{\epsilon h}\right]$.

In particular,

(c) The number of $\Lambda$-orbits on $\left[\tilde{X}_{\epsilon h}\right]$ is equal to the order of $W$.

A result closely related to (c) (but not (c) itself) appears in [4].

6. Let [S] be the set of irreducible components of $\mathfrak{S}$ (a finite set naturally indexed by $W$ by $w \mapsto \overline{\mathfrak{S}_{w}}$ ). The bijection $5(\mathrm{~b})$ gives rise to an imbedding $[\mathfrak{S}] \rightarrow\left[\tilde{X}_{\epsilon h}\right], \overline{\mathfrak{S}_{w}} \mapsto \overline{\tilde{\Omega}_{w, 1}}$ hence to an imbedding of vector spaces

(a) $\mathbf{C}[\mathfrak{S}] \rightarrow \mathbf{C}\left[\tilde{X}_{\epsilon h}\right]$

with bases $[\mathfrak{S}],\left[\tilde{X}_{\epsilon h}\right]$. Springer has shown that $W$ acts naturally on $\mathbf{C}[\mathfrak{S}]$ (this is known to be the regular representation of $W$ in a nonstandard basis). In [3] it is shown that the affine Weyl group of $G$ acts naturally on $\mathbf{C}\left[\tilde{X}_{\epsilon h}\right]$. Hence, by restriction, $W$ acts on $\mathbf{C}\left[\tilde{X}_{\epsilon h}\right]$. From the definitions we see that the imbedding (a) is compatible with the $W$-actions.

\section{References}

1. D. Kazhdan and G. Lusztig, Fixed point varieties on affine flag manifolds, Isr. J. Math., 162 (1988), 129-168. 
2. G. Lusztig, Singularities, character formulas and a $q$-analog of weight multiplicities, Astérisque, 101-102 (1983), 208-229.

3. G. Lusztig, Affine Weyl groups and conjugacy classes in Weyl groups, Transform. Groups, (1996), 83-97.

4. C. C. Tsai, Components of affine Springer fibres, arxiv:1609.05176. 